\title{
The Elephant and the Mouse: making a large department feel small and a small department feel large.
}

\begin{abstract}
A. -
Engineering Education in Canada is carried out at 44 currently accredited university programs. Collectively, the system graduates more than 12,000 new engineers each year. One quarter of those students study at a large schools having more than 1000 students in each yearly cohort. Many more study in medium-sized classes having more than 300 students each year. There also campuses where an engineering class is fewer than 30 students. How does the student experience differ from the opposite ends of this size spectrum?

The paper compares aspects of the student experience at one of the largest programs in the country with the student experience at one of the smallest. Based upon experience teaching at both scales, and upon curriculum revision at both scales, the issues relevant at each scale of program will be discussed. The paper will compare both the positive and the negative perceptions of program scale. For example; students in the large scale experience a negative sense of "being just a number", versus the rewarding sense of being "in the big leagues" where technological advances continue to be made. At the small scale, the corresponding negative perception by students is they may be limited in opportunities, versus their positive impact of feeling nurtured by personal attention from staff and faculty. These and other issues will be examined in the paper.

The paper will draw upon student surveys of attitude carried out in both a large and a small engineering campus. In addition to survey results, student focus group discussions will be presented which give a deeper sense of the student experience versus scale. In particular, the work presented will give comparison of the theoretical/hands-on opportunities at each of the two programs compared with the depth of learning opportunities in engineering science that students encounter in each setting.

The conclusion drawn is one of fit rather than "better" or "worse". For some students, their needs may be best served by the values in a large institution, while some students find better success in a smaller one. Whilst the details for every student are complex and personal, the aim in this paper is to attempt to explain the relevance of the whole spectrum of program scale. The paper concludes with a proposed map of student needs and skills indicating an optimal match with one or the other university scale.
\end{abstract}

Education research 